\title{
Unusual Swelling in the Head and Neck Region
}

\author{
Vipasha Yogi', Shruti Baruah ${ }^{2}$, Pratibha Vyas ${ }^{3}$, Kunal Sharma ${ }^{4}$
}

\begin{abstract}
These are case series with six patients who presented unusual neck swelling. Worldwide, the most common swelling in the neck region is a enlargement of cervical lymph nodes. Each country has a different etiology and geographical distribution for cervical lymphadenopathy. Tuberculosis, metastatic cancers, and lymphomas are the commonest causes. However, in clinical practice, we encounter rare neck swelling. Such swellings are due to their less common and unusual presentation, and its clinical diagnosis is rarely attained. In such cases, proper diagnosis and management are required to attain the desired results.

Materials and methods: In our tertiary healthcare center, we encountered such case in ENT OPD. Five cases were evaluated in this article along with their management. Such rare causes of neck swellings are therapeutically challenging, as they cause diagnostic dilemmas.

Results: Histopathology and immunohistochemistry were the mainstay of diagnosis, and surgery was curative in most cases.

Keywords: Cysticercosis, Fibroma tongue, Filariasis, Mucoepidermoid carcinoma palate, Neck swellings, Plunging ranula.

Journal of Mahatma Gandhi University of Medical Sciences \& Technology (2019): 10.5005/jp-journals-10057-0098
\end{abstract}

\section{INTRODUCTION}

Neck swellings are common complain that present in all age-groups from various etiology, ranging from congenital to acquired, from cysts, inflammatory, infective to neoplastic disease, encompassing any neck structure. To aid with the correct diagnosis for appropriate management, all clinicians should understand the embryology and anatomy. Knowledge of patient's age, associated symptoms, and anatomical location of the lump, according to duration acute, subacute or chronic swelling, is key to proceeding to treatment generally, or they are an indication for referral to further investigations, including imaging and surgery.

Few rare cases of head and neck region swellings are discussed with unusual site of respective disease presentation with appropriate management.

\section{Case Descriptions}

\section{Case 1}

A 47-year-old male presented to ENT OPD of Mahatama Gandhi Hospital with complains of slow-growing swelling, of approximately 2 years duration, involving hard palate on the right side. The swelling was asymptomatic with no complains of pain and bleeding. He had not undergone any surgeries of head and neck and had no complaints of pharyngeal or airway obstruction. On general physical examination, the patient was a well-oriented and a moderately built individual with no signs of any systemic illness. The clinical examination revealed nonulcerated, dome-shaped, palatal swelling on his hard palate. The mass had a bosselated surface, and the overlying mucosa was normal. The lesion was smooth, firm, and nontender on palpation of size approximately $2 \times 1 \mathrm{~cm}$. All preoperative blood, and urine investigations were done, and results were within normal limits. Nasal intubation was done and excision of the mass was carried out. The excised mass of $1 \times 1 \times 1.5-\mathrm{cm}$ dimensions was sent for histopathological examination. Following excision of tumor, surgical wound was closed. Histopathological examination shows squamous epithelium with underlying mucus gland and fobroadipose tissue. There is a malignant epithelial tumor showing
1,3,4 Department of ENT and Head and Neck Surgery, Mahatma Gandhi Medical College and Hospital, Mahatma Gandhi University of Medical Sciences and Technology, Jaipur, Rajasthan, India

${ }^{2}$ Deptartment of ENT, Dispur Polyclinic and Multispeciality Hospital, Guwahati, Assam, India

Corresponding Author: Pratibha Vyas, Department of ENT and Head and Neck Surgery, Mahatma Gandhi Medical College and Hospital, Mahatma Gandhi University of Medical Sciences and Technology, Jaipur, Rajasthan, India, Phone: +91 9462815334, e-mail: drpratibhaV92@gmail.com

How to cite this article: Yogi V, Baruah S, Vyas P, et al. Unusual Swelling in the Head and Neck Region. J Mahatma Gandhi Univ Med Sci Tech 2019;4(1):7-13.

Source of support: Nil

Conflict of interest: None

squamous epithelium and glandular differentiation with mucinsecreting cells and pools of mucin overall features suggesting a low-grade mucoepidermoid carcinoma (Figs 1 and 2). For further management in the form of Chemotherapy, the patient was referred to Medical Oncology. Postoperative contrast-enhanced computed tomography (CECT) scan (Fig. 3) of head and neck region was done, and no obvious residual lesion was seen on the hard palate (Fig. 4). Nasopharynx, oropharynx, and larngopharynx were normal with no bony erosion.

\section{Case 2}

A 34-year-old male patient came to ENT OPD with complain of swelling on the left side neck for past 6 months. The swelling gradually progressed in size, with no associated pain or history of fever, throat pain, or cough of long duration. No similar swelling was present in others parts of the body. On clinical examination, two swellings were palpable on the left cervical $2 a$ and $2 b$ measuring $2 \times$ 3 and $1 \times 2 \mathrm{~cm}$, respectively, mobile, smooth, firm in consistency, and tender with normal skin overlying the swellings. Ultrasound of the neck (Fig. 5) showed two cystic lesions in substance of left sternocleidomastoid, the larger one measuring approximately $28 \times$ 


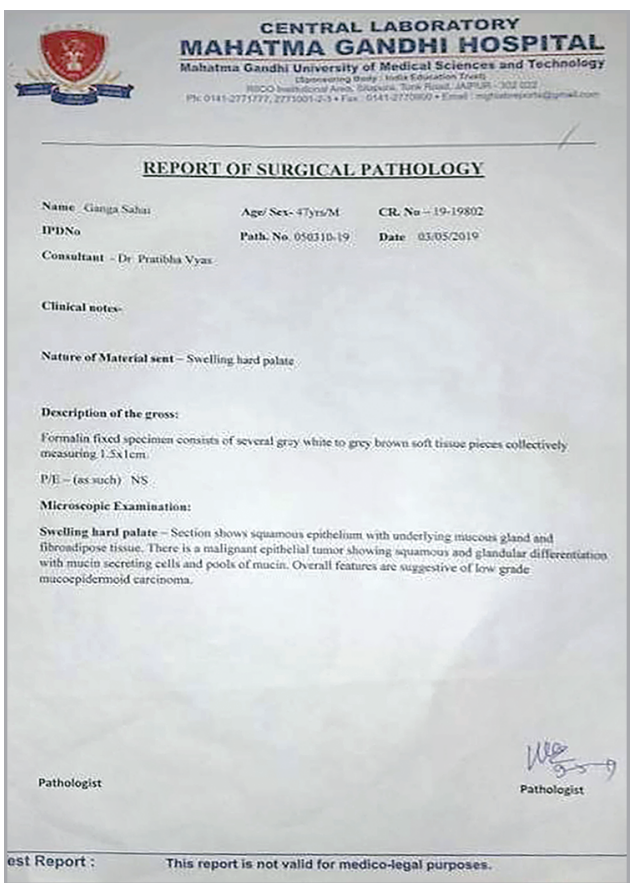

Fig. 1: Histopathology report

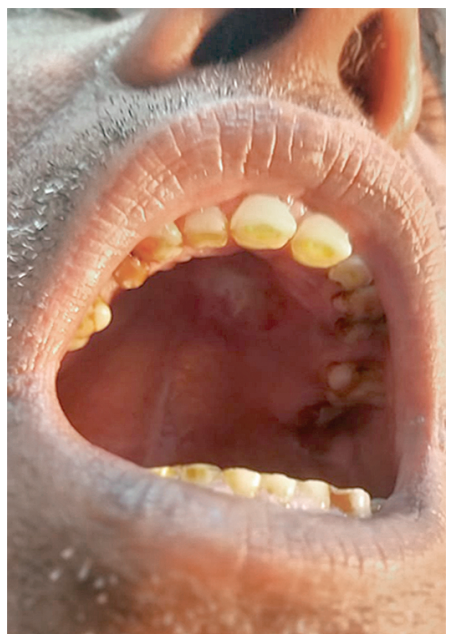

Fig. 3: Postoperative site of excision

$11 \mathrm{~mm}$. The smaller one shows small cyst with the cyst appearance measuring approximately $14 \times 6 \mathrm{~mm}$ with no internal vascularity. Fine-needle aspiration cytology (FNAC) of left cervical swelling was suggestive of cystecercosis. After complete workup, the case was taken for surgery under general anesthesia. Horizontal skin incision was given, and both swellings were excised (Figs 6 and 7) after dissection of soft tissue, and wound closure was done in two layers. The excised specimens (Fig. 8) were sent for histopathological study which showed features suggestive of cysticercosis.

\section{Case 3}

A 14-year-old female patient presented to the ENT OPD of Mahatma Gandhi Hospital, Jaipur, with complaints of swelling on the left side of the neck which had developed over a duration of 9 months. The swelling gradually progressed in size, with no associated pain, or history of fever, throat pain, or cough of long duration. The patient's medical history was noncontributory. There was no

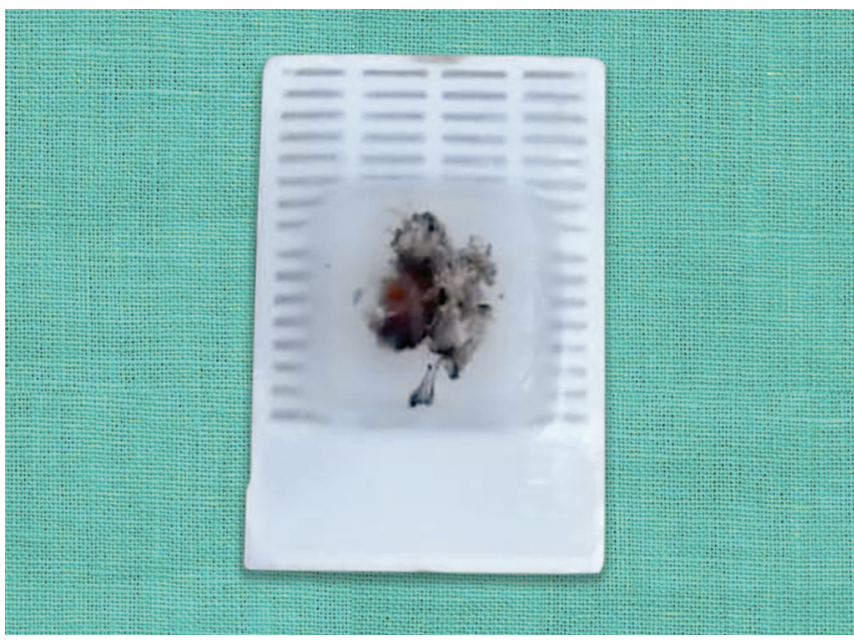

Fig. 2: Histopathology specimen

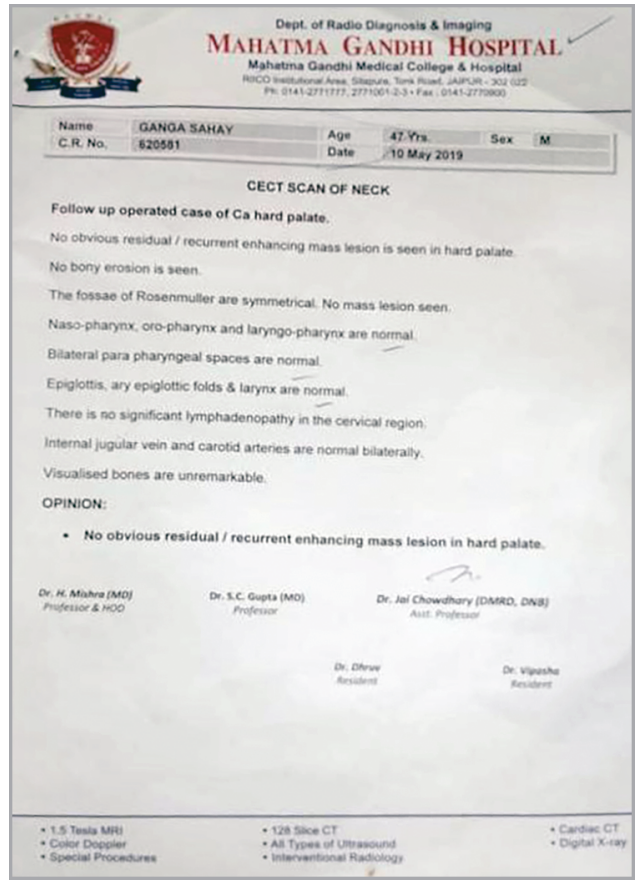

Fig. 4: Postoperative contrast-enhanced computed tomography scan

similar swelling in other body parts with no previous history of similar lesions in past. On clinical examination, two swellings were palpable on the left cervical region at the level of $2 a$ and $2 b$ lymph nodes measuring, $3 \times 2 \mathrm{~cm}$ and $2 \times 2 \mathrm{~cm}$, respectively, smooth, firm in consistency, mobile, and nontender with normal skin overlying the swellings. Ultrasound of the neck showed multiple cervical lymphadenopathy at left cervical region. Fine-needle aspiration cytology of the right upper cervical lymph node was suggestive of filariasis. After complete workup, the case was taken for surgery under general anesthesia. Horizontal skin incision was given, and both swellings were excised after dissection of soft tissue, and wound closure was done in two layers. The excised specimens (Fig. 9) were sent for histopathological study (Fig. 10) which showed features suggestive of chronic inflammatory cells with eosnophils. The tissue shows several parasites, and morphology of parasites is suggestive of filariasis (Fig. 11). 


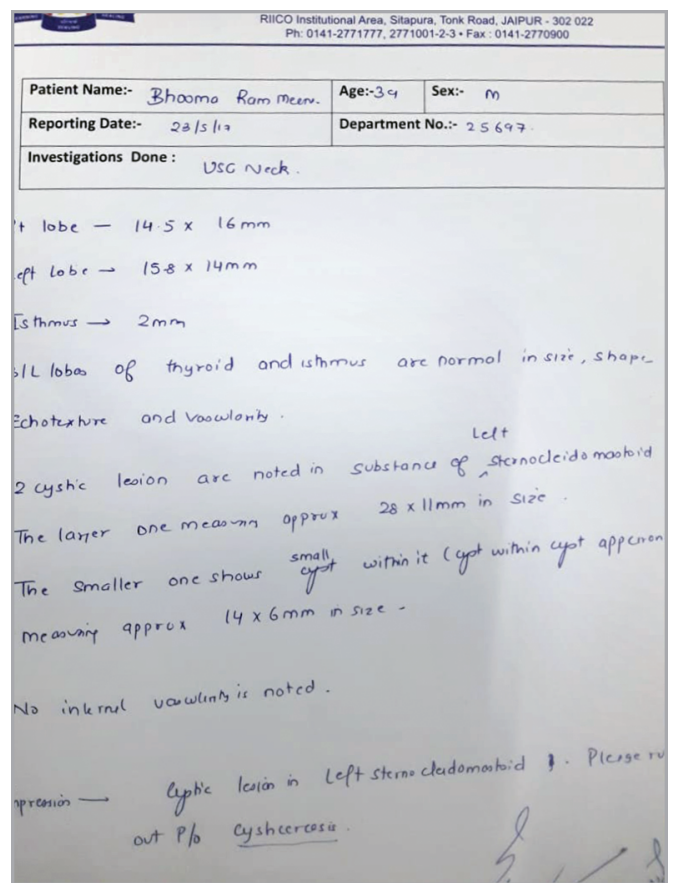

Fig. 5: Ultrasonography neck swelling

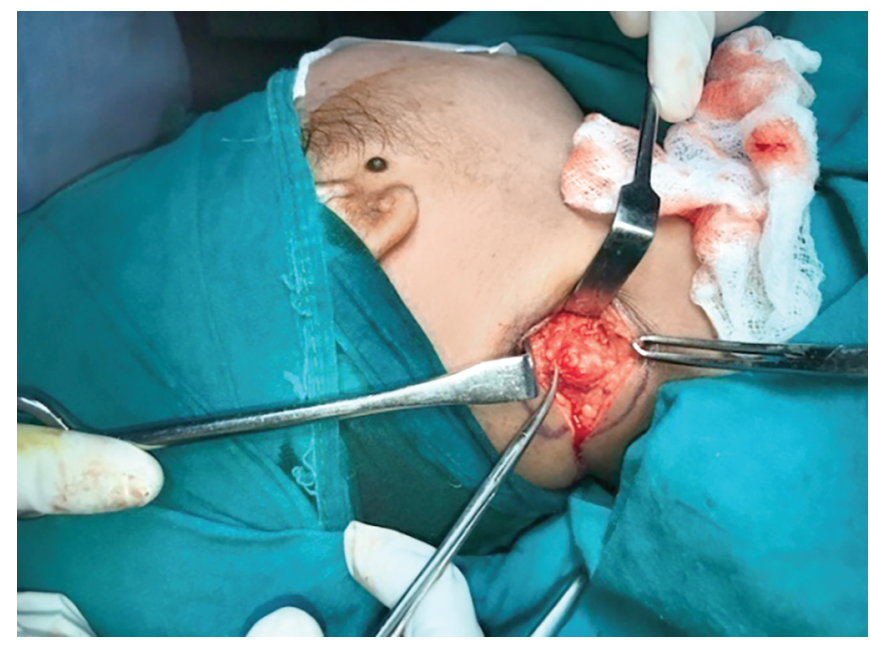

Fig. 7: Excision of lesion

\section{Case 4}

A 50-year-old male patient came to ENT OPD with complain of swelling tongue since 1 year. Swelling was slow growing with no complain of bleeding and pain. Swelling was not associated with difficulty in swallowing or breathing. No associated weight loss or fever was present. On clinical examination, approximately $5 \times 6 \mathrm{~cm}$-sized fixed tender swelling was seen arising from the right lateral border tongue with no ulceration bleeding. Other parts of nasopharynx, oropharynx, and laryngopharynx was normal on endoscopic examination. No associated cervical lymphadenopathy was seen. Preoperative workup under general anesthesia was done to excise the swelling and closure was done. Fibroma of the tongue was diagnosed on histopathologial examination.

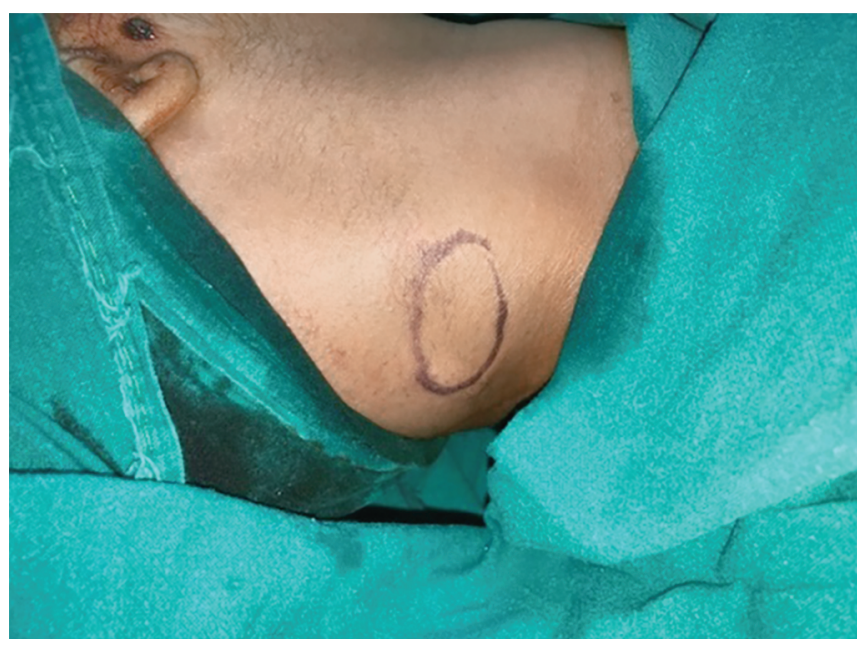

Fig. 6: Marking the lesion site

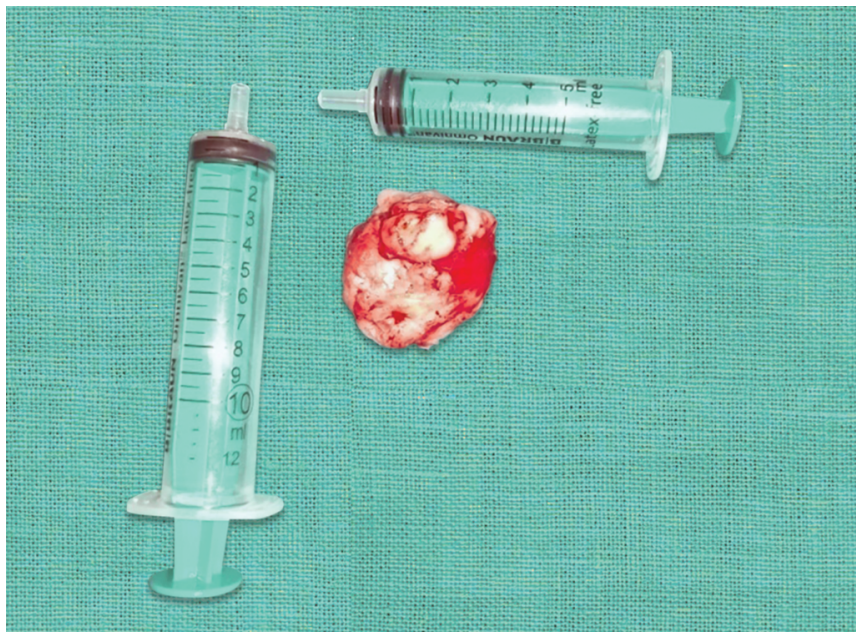

Fig. 8: Excised specimen

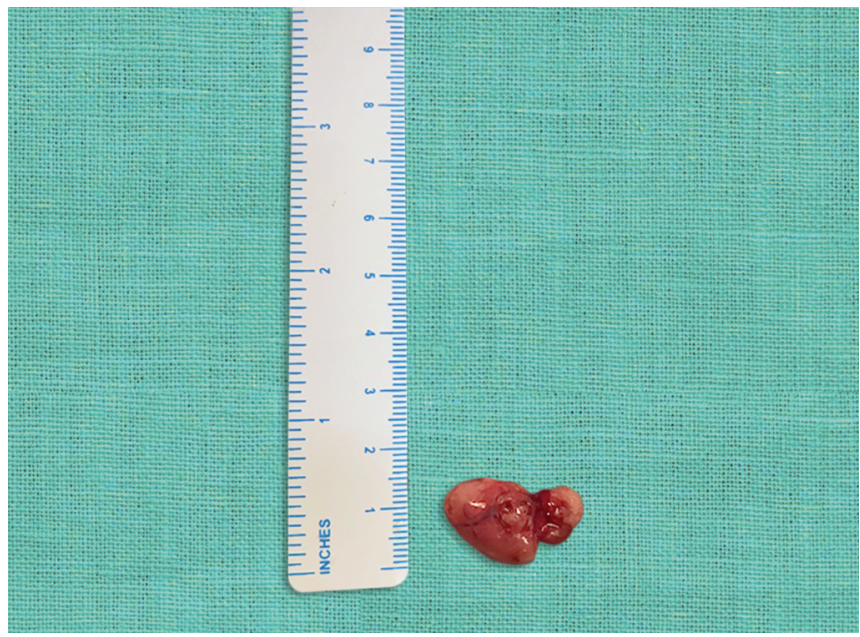

Fig. 9: Excised specimen

\section{Case 5}

A 13-year-old male child came to ENT OPD with complain of swelling in the left cervical region for the last 3 months. Swelling 


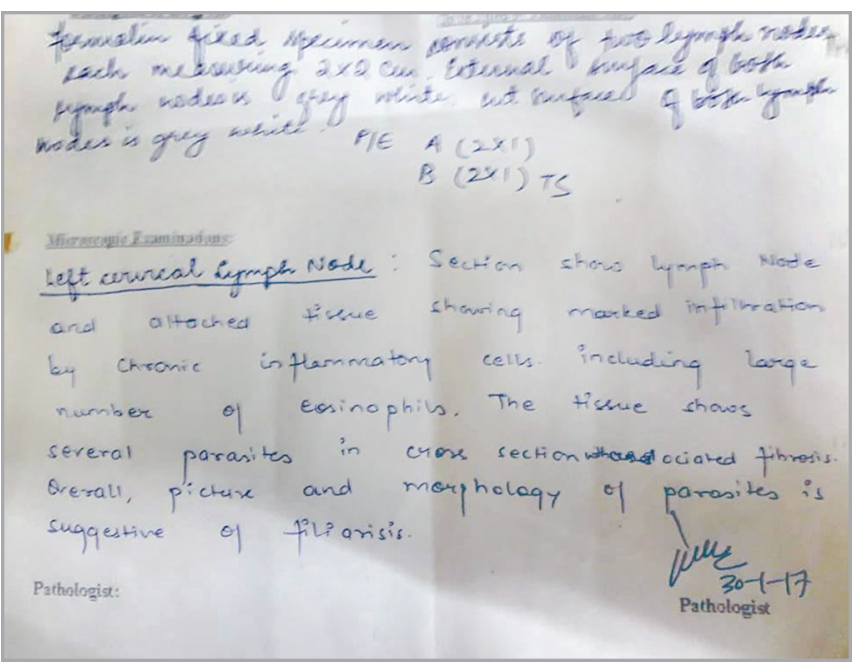

Fig. 10: Histopathologic examination report s/o filariasis

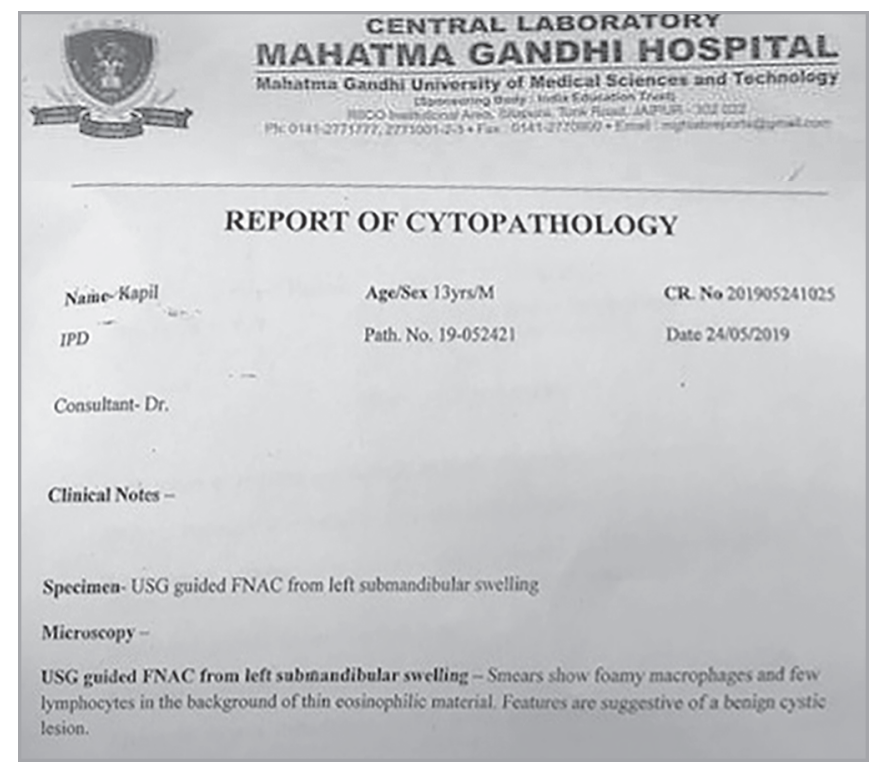

Fig. 12: Fine-needle aspiration cytology report

was progressive and painless with no complain of difficulty in swallowing and breathing. On examination $2 \times 3 \times 3-\mathrm{cm}$ mobile firm swelling was palpable. No intraoral extension was seen in the floor of mouth. Swelling was irreducible on coughing and blowing. Medical history was not significant. On FNAC (Fig. 12), the features were suggestive of benign cystic lesion. On CECT of the neck (Figs 13 and 14), a multi-septated cystic lesion of measuring $23 \times$ $26 \times 27 \mathrm{~mm}$ was seen in the left submadibular region. The lesion is abutting the submandibular gland not involving it. Adjacent mandible is not eroded. Multiple cervical lymphadenopathy was seen. Preoperative workup was done, and the patient was taken for surgery under general anesthesia. Horizontal incision was given in left submandibular region. Swelling was traced through out the course of cystic swelling, and enucleation was done (Fig. 15). Closure was done into two layers. On histopathological examination plunging ranula was diagnosed.

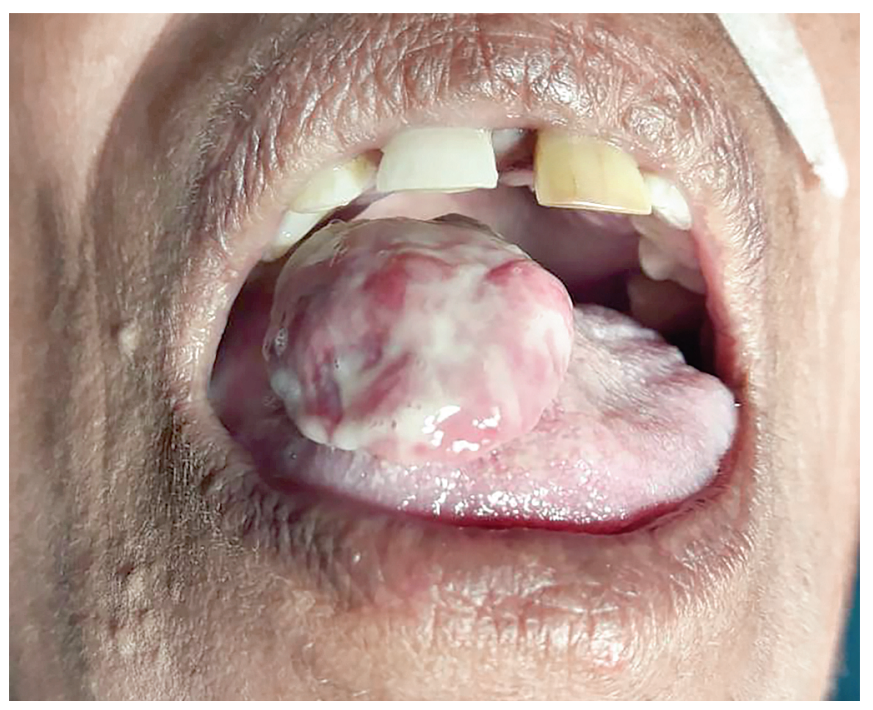

Fig. 11: Clinical picture of tongue swelling at right lateral border tongue

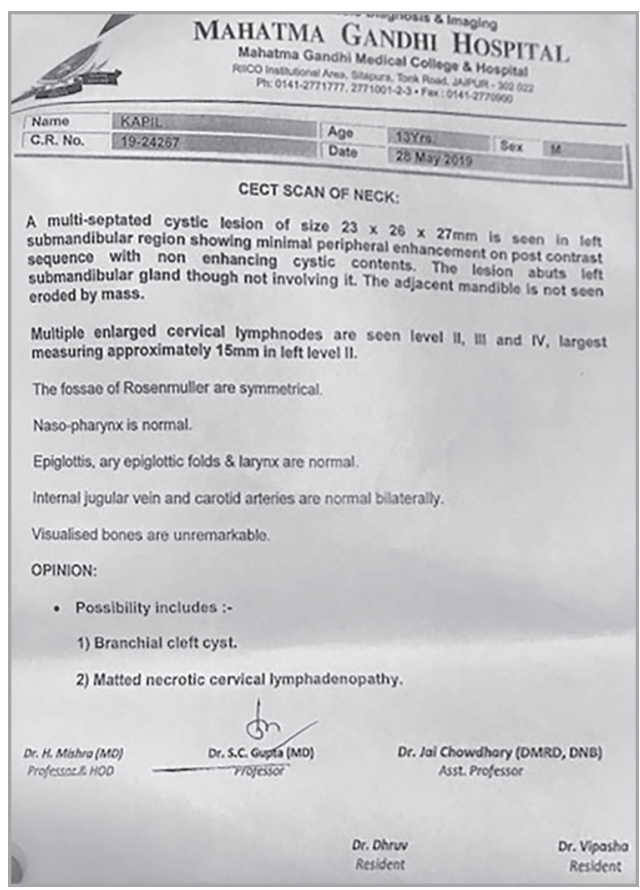

Fig. 13: contrast-enhanced computed tomography scan neck

\section{Discussion}

Diagnostic dilemma of head and neck swellings needs systematic evaluation. Fine-needle aspiration cytology is the standard first investigation in case of neck swellings; but sometimes, FNAC fails to diagnose the the rare diseases of any of the neck masses due to inadequate material or procedure. To make a treatment plan for such cases, proper evaluation with various imaging modalities such as, CECT, magnetic resonance imaging (MRI), and ulstrasound (USG), are required. In this article, we presented a series of uncommon swellings in the head and neck region that we encountered in our hospital, which is a tertiary healthcare institute. 


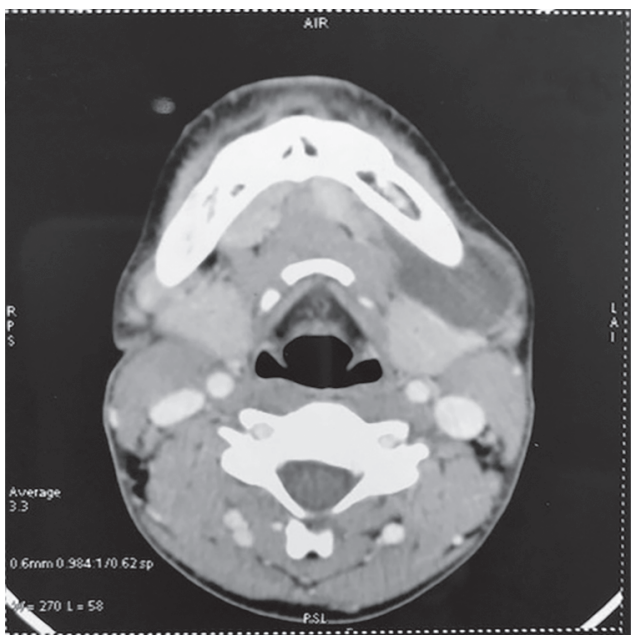

Fig. 14: Contrast-enhanced computed tomography showing extent of swelling

\section{Giant Cell Fibroma}

Giant cell fibroma (GCF) was described as a fibrous hyperplastic soft tissue. It appears that stimulus from an unexplained origin can have a role in its etiology. GCF was first described by Weathers and Calliham as a new entity of fibrous hyperplastic soft tissue in 1974. ${ }^{1,2}$ GCF is a rare fibrous lesion, and a very few case reports are available regarding this lesion. It represents 2 to $5 \%$ of all biopsied fibrous lesions of oral cavity. ${ }^{3}$ There is controversy about the origin of GCF. ${ }^{2}$ GCF occurs commonly in patients under the age of 30 years with a slight female predilection. ${ }^{1}$

Gingiva followed by the tongue and buccal mucosas are the most involved locations. ${ }^{4}$ It usually presents as a pedunculated or sessile asymptomatic exophytic lesion with size of less than $1 \mathrm{~cm}$ in the oral cavity. Irritation fibroma is a reactive fibrous proliferation but with some discriminative differences such as nonsmoth surface texture, Histopathologically, GCF consists avascular collagenic stroma of multinucleated fibroblasts in close relation to the overlaying epithelium. These fibroblasts have oval-shaped nuclei within the eosinophilic cytoplasm resembling a stellate appearance. ${ }^{2,5,6}$ Positive reactivity for vimentin (but not for cytokeratin, neurofilament, HHF, CD 68, HLA DR, tryptase and S100) suggests a fibroblastic phenotype. ${ }^{3}$ It may be misdiagnosed as irritation fibroma, especially in cases where it is on the buccal mucosa, the most common location for fibroma. Accurate diagnosis is based on biopsy with clinical examination to see surface texture roughness ${ }^{1}$

\section{Plunging Ranula}

A ranula by definition is a mucus-filled cavity, a mucocele, in the floor of the mouth in relation to the sublingual gland. ${ }^{7,8}$ Ranulas are characteristically large $(>2 \mathrm{~cm})$ and present as a tense fluctuant dome-shaped vesicle, sometimes with a blue hue. The most common site is floor of the oral cavity laterally of frenulum not crossing midline. Plunging ranula, a clinical variant with moderate incidence, occurs when the mucin fluid pressure plunges through a perforation in the mylohyoid muscle in the submandibular space. ${ }^{9,10}$ Prevalence of about 0.2 per 1,000 cases accounts for $6 \%$ of all oral sialocysts. The number of ranulas that represents a true retention cyst ranges from less than $1 \%$ to $10 \% .{ }^{11}$ Ranulas usually occur in children and young adults, with peak in the second decade. ${ }^{11}$ The cervical variant occurs later in the third decade. Plunging ranulas

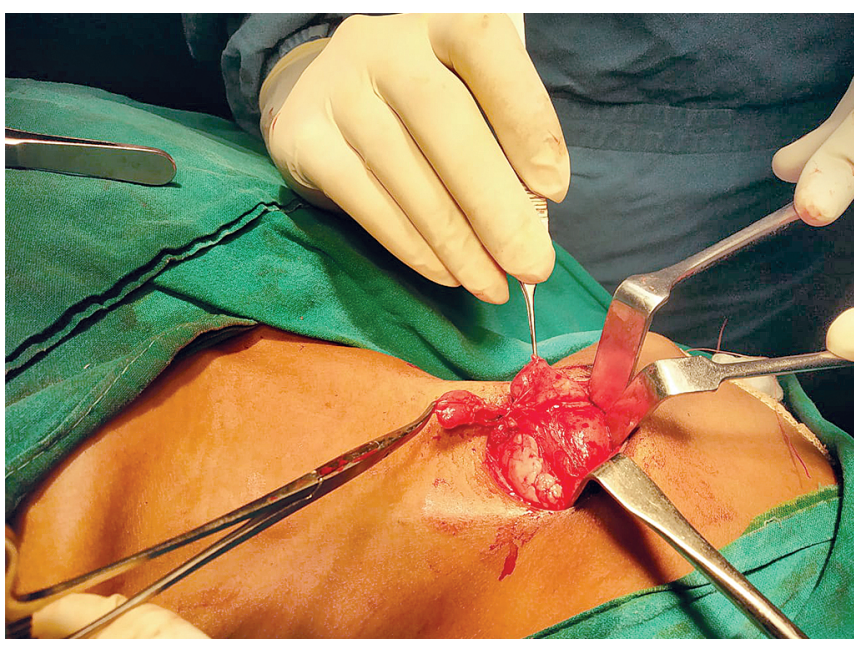

Fig. 15: Excised specimen s/o of cystic consistency

arise in the neck by following four mechanisms: (a) The sublingual salivary gland may dissect through the mylohyoid, or an ectopic sublingual gland may exist on the cervical side of mylohyoid. (b) A dehiscence or hiatus in the mylohyoid muscle may occur along the lateral aspect of the anterior two-thirds of the muscle. (c) $45 \%$ of plunging ranulas occur iatrogenically after surgical remove oral ranulas. (d) A duct from the sublingual salivary gland may join the submandibular salivary gland or its duct which allows ranulas to form in continuity with the submandibular salivary gland. Therefore, the ranula accesses the neck from behind the mylohyoid muscle. USG, CT SCAN, and MRI (most sensitive) are to be one to confirm the diagnosis and see the extent of involvement. Treatment includes surgical excision by cervical and intra-oral routes with or without excision and drainage of part of salivery gland depending upon the extent of the disease. ${ }^{12}$ Other options include cryosurgery, marsupilization with or with out cauterization of the lesion linning, ${ }^{11} \mathrm{CO}_{2}$ laser vaporize of ranula, ${ }^{13}$ rarely radiation therapy, and intracystic injection of streptococcal preparation. Excision of the ranula with the associated sublingual salivary gland is the most accepted method with low recurrence rate. ${ }^{11}$ Risk of paresis and paralysis of the marginal mandibular nerve is the most common complication following surgical therapy of ranula. ${ }^{14}$

\section{Cysticercosis}

Taenia solium, a potentially dangerous parasite, causes human cysticercosis. Humans acquire the infection through consumption of undercooked pork, raw vegetables, or by drinking water contaminated by this parasite. ${ }^{15}$ Cysticercosis can cause a gamut of manifestations affecting the central nervous system (CNS), skeletal muscles, heart, eyes, and subcutaneous tissues. ${ }^{16}$ These palpable nodules are usually misdiagnosed for lymph nodes or benign tumors, such as lipoma, neurofibroma, or epidermal inclusion cysts. ${ }^{17,18}$ It can affect the CNS causing lethal complications or more commonly present with superficial palpable lesions amenable to FNAC. ${ }^{15}$ Aspiration of the lesion usually yields a clear fluid, which is a strong pointer to the diagnosis ${ }^{17,19}$ and varies from clear to mucoid usually. The parasitic fragments comprise bluish fibrillary structures that are part of the bladder wall and contain tiny parasitic nuclei that appear dot-like. ${ }^{19}$ Rarely it can be found as detached hooklets and/or calcareous corpuscles. Cysticercosis in children is relatively rare compared to adults. Second most common site after CNS is skeletal muscles. When the larva dies, 
it induces an aggressive granulomatous inflammatory response, leading the characteristic organ-specific symptoms. Biopsy from the lesion is gold standard in proving a definitive diagnosis. HPE confirms the diagnosis by showing scolices, hooklets, and cyst wall. Treatment of cysticercosis is by orally administered Albendazole or Praziquantel, while ophthalmic cysticercosis requires surgery before medical treatment is started. All family members should be screened for infection or empirically treated with Albendazole or Praziquantal. ${ }^{20}$

\section{Filaria}

Lymphatic filariasis is an endemic disease in the tropical countries and is the most common cause of acquired lymphedema in the world. Wuchereria bancrofti is the main and important etiological agent. While the presentation of filariasis in limbs is common, isolated presentation as a single enlarged lymph node in the neck is very rare. ${ }^{21}$ In tropical and subtropical countries, filariasis is a major health issue. Over 1 billion people are at risk, with more than 1 million people being already infected. ${ }^{22}$ In the early stages of filarial disease of the neck, medical therapy with DEC can produce excellent results. Results of domestic lymphatic filariasis show that chronic lymphadenopathy in the head and neck region is the most common presenting symptoms. Accurate diagnosis is made after surgical lymph node excision. Rarely patients have circulating microfilariae in body, so surgical excision of the affected lymph node is often curative, and antihelminth treatment post excision can be withheld if patients remain asymptomatic.

In endemic regions, the current treatment consists of diethylcarbamazine (DEC) $6 \mathrm{mg} / \mathrm{kg} /$ day divided into three doses. ${ }^{23} \mathrm{It}$ is typically given as a 12-day course; however, a single dose may be just as effective. ${ }^{23-25}$ Some authors advocate for the addition of 4 - to 6-week course of doxycycline $200 \mathrm{mg} /$ day, which targets Wolbachia, the intracellular bacterial symbiont of Wuchereria bancrofti and Brugia spp. ${ }^{24,26}$ Ivermectin, given as a single 200- or 400- $\mu \mathrm{g} / \mathrm{kg}$ dose, has an efficacy similar to DEC and can be used as a monotherapy or in combination with DEC to augment its microfilarial activity. ${ }^{25,27}$

\section{Mucoepidermoid Carcinoma Palate}

Mucoepidermoid carcinoma (MEC) is the epithelial salivary gland neoplasm of the oral cavity. It accounts for $<3 \%$ of all head and neck tumors. It occurs in about $5 \%$ of patients younger than 18 -year-old, mostly affecting women. It is composed of mucusproducing, squamous, and intermediate-type cells. Of all cases, about two-thirds arise within the parotid gland, and other onethird arises within the minor salivary glands. MEC of minor salivary glands can be located on the palate, retromolar area, floor of the mouth, lips, and tongue, buccal mucosa and rarely presents as primary jaw tumor, laryngeal, lacrimal, nasal, paranasal, tracheal, or pulmonary tumor. Adults during the fifth and sixth decades are most frequently affected. ${ }^{28}$ Although rare, it is the main malignant salivary gland tumor in children, particularly adolescents, and affects women more than men. Histpathologically, MECs are classified as low-, intermediate-, and high grade. The low-grade variety commonly develops a nesting pattern with multiple wellcircumscribed squamous nests containing numerous clear cells. Intermediate-variety tumors are less cystic with greater tendency to form large sheets of squamous cells and often shows more prominent intermediate cell population. High-grade tumors are predominantly solid and show greater degrees of atypia. Clinically, MEC of palatal region presents as firm, painless swellings and may mimic mucoceles or vascular lesions. MEC of the palate follows a wide surgical excision with adjacent free margins. ${ }^{29}$ If there is no evidence of bony involvement, the tumor should be dissected down to the periosteum. If there is any evidence of periosteal involvement or bone erosion, removal of the involving bone is indicated. If the lesion is restricted to the alveolar region, alveolectomy is performed. This consists of removal of the affected alveolus and a limited portion of the maxilla. Failure to detect lesion in its early stage leads to involvement of overlying maxillary sinus and the nasal cavity, requiring more extensive surgery including palatectomy, infrastructure maxillectomy, or extended maxillectomies. Such procedures often require an extraoral approach and need for further reconstructive surgery of the oral defect. ${ }^{30}$ High-grade tumors require more aggressive surgical approach with postoperative radiotherapy and chemotherapy. Low- to intermediate-grade MECs originating from intraoral minor salivary glands have a very low recurrence rate $(<10 \%)$ and a high survival rate (90\%). Low- and intermediate-grade MECs have an indolent clinical course and a rare chance for metastasis. Radical neck dissection is indicated if clinical evidence of metastasis is present. Prognosis depends on grade and stage of tumor. A close clinical follow-up should be done for lifetime because low- and intermediate-grade MEC in this age-group can recur many years late..$^{31,32}$

\section{References}

1. Bagheri F, Rahmani S, Azimi S, et al. Giant cell fibroma of the buccal mucosa with laser excision: report of unusual case. Iran J Pathol 2015;10(4):314-317.

2. Sabarinath B, Sivaramakrishnan M, Sivapathasundharam B. Giant cell fibroma: a clinicopathological study. J Oral Maxillofac Pathol 2012;16(3):359-362. DOI: 10.4103/0973-029X.102485.

3. Jimson S, Jimson S. Giant cell fibroma: a case report with immunohistochemical markers. J Clin Diagn Res 2013;7(12): 3079-3080. DOI: 10.7860/JCDR/2013/6476.3859.

4. Kuo RC, Wang YP, Chen HM, et al. Clinicopathological study of oral giant cell fibromas. J Formos Med Assoc 2009;108(9):725-729. DOI: 10.1016/S0929-6646(09)60396-X.

5. Bakos LH. The giant cell fibroma: a review of 116 cases. Ann Dent 1992;51(1):32-35.

6. Weathers DR, Campbell WG. Ultrastructure of the giant cell fibroma of the oral mucosa. Oral Surg Oral Med Oral Pathol 1974;38(4):550-561. DOI: 10.1016/0030-4220(74)90086-3.

7. Baurmash HD. Mucoceles and ranulas. J Oral Maxillofac Surg 2003;61(3):369-378. DOI: 10.1053/joms.2003.50074.

8. Neville BD, Damm DD, Allen CM, et al. Oral and Maxillofacial Pathology. 2nd ed., Philadelphia, PA, USA: Saunders; 2002.

9. Langlois NEl, Kolhe P. Plunging ranula: a case report and a literature review. Hum Pathol 1992;23(11):1306-1308. DOI: 10.1016/00468177(92)90300-r.

10. Skouteris CA, Sotereanos GC. Plunging ranula: report of a case. J Oral Maxillofac Surg 1987;45(12):1068-1072. DOI: 10.1016/02782391(87)90167-4.

11. Gupta A, Karjodkar FR. Plunging ranula: a case report. ISRN Dent 2011;2011:806928. DOI: 10.5402/2011/806928.

12. Yoshimura Y, Obara S, Kondoh T, et al. A comparison of three methods used for treatment of ranula. J Oral Maxillofac Surg 1995;53(3): 280-283. DOI: 10.1016/0278-2391(95)90224-4.

13. Mintz S, Barak S, Horowitz I. Carbon dioxide laser excision and vaporization of nonplunging ranulas: a comparison of two treatment protocols. J Oral Maxillofac Surg 1994;52(4):370-372. DOI: 10.1016/0278-2391(94)90439-1.

14. Zhao Y-F, Jia J, Jia Y. Complications associated with surgical management of ranulas. J Oral Maxillofac Surg 2005;63(1)):51-54. DOI: 10.1016/j.joms.2004.02.018. 
15. Jashnani Kusum D, Desai Heena $M$, Shetty Jyothi B, et al. Fine-needle aspiration cytology of subcutaneous cysticercosis: a series of five cases. Annals of Tropical Medicine and Public Health 2016;9(1):73. DOI: 10.4103/1755-6783.168695.

16. Chatterjee KD. Phylum platyhelminthes: class cestoidea Chatterjee KD, ed. Parasitology (Protozoology and Helminthology). 13th ed., New Delhi: Thomson Press; 2011. pp. 143-171.

17. Suchitha $S$, Vani $K$, Sunila R, et al. Fine needle aspiration cytology of cytsicercosis-a case report. Case Report Infectious Disease 2012;2012:854704. DOI: 10.1155/2012/854704.

18. Elhence P, Bansal R, Sharma S, et al. Cysticercosis presenting as cervical lymphadenopathy: a rare presentation in two cases with review of literature. Niger J Clin Pract 2012;15(3):361-363. DOI: 10.4103/11193077.100652.

19. Goyal P, Sehgal S, Ghosh S, et al. A cytological study of palpable superficial nodules of parasitic origin: a study of 41 cases. Patholog Res Int 2014;2014:373472. DOI: 10.1155/2014/373472.

20. Kalra V, Seth R, Mishra D. Extraneural cysticercosis - presenting as painless cervical swellings. J Trop Pediatr 2006;52(2):141-143. DOI: 10.1093/tropej/fmi090.

21. Raveendran R. Lymph node enlargement in neck filariasis as a rare cause: a case report. Iran J Med Sci 2017;42(5):493-496.

22. Lanham A, Mwanri L. The curse of lymphatic filariasis: would the continual use of diethylcarbamazine eliminate this scourge in papua new guinea? Am J Infect Dis Micro 2013;1:5-12. DOI: 10.12691/ajidm1-1-2.

23. Goel TC, Goel A. Treatment and prognosis Goel TC, Goel A, ed. Lymphatic Filariasis. Singapore: Springer Singapore; 2016. pp. 81-85.
24. Taylor MJ, Hoerauf A, Bockarie M. Lymphatic filariasis and onchocerciasis. Lancet 2010;376(9747):1175-1185. DOI: 10.1016/S01406736(10)60586-7.

25. Dreyer G, Coutinho A, Miranda D, et al. Treatment of bancroftian filariasis in Recife, Brazil: a two-year comparative study of the efficacy of single treatments with ivermectin or diethylcarbamazine. Trans R Soc Trop Med Hyg 1995;89(1):98-102. DOI: 10.1016/00359203(95)90674-6.

26. Goel TC, Goel A. Antifilarial drugs. Goel TC, Goel A, ed. Lymphatic Filariasis. Singapore: Springer Singapore; 2016.

27. Greene BM, Taylor HR, Cupp EW, et al. Comparison of ivermectin and diethylcarbamazine in the treatment of onchocerciasis. 1985;313(3):133-138. DOI: 10.1056/NEJM198507183130301.

28. Jarde SJ, Das S, Narayanswamy SA, et al. Mucoepidermoid carcinoma of the palate: a rare case report. J Indian Soc Periodontol 2016;20(2):203-206. DOI: 10.4103/0972-124X.170828.

29. Mathew AL, Joseph BB, Sarojini DM, et al. Mucoepidermoid carcinoma of palate - a rare entity. Clin Pract 2017;7(4):1009. DOI: 10.4081/ cp.2017.1009.

30. Munhoz Ede A, Cardoso CL, Tjioe KC, et al. Atypical clinical manifestation of Mucoepidermoid carcinoma in the palate. Gen Dent 2009;57(6):e51-e53.

31. Moraes P, Pereira C, Almeida O, et al. Paediatric intraoral mucoepidermoid carcinoma mimicking a bone lesion. Int J Paediatr Dent 2007;17(2):151-154. DOI: 10.1111/j.1365-263X.2006. 00770.x.

32. Sharanjeet G, Amit M, Shivani A, et al. Mucoepidermoid carcinoma of hard palate. 2018;61(3):397-398. 